it can be depended on for counts extending over a long period.

A detailed discussion will be published elsewhere (Z. tech. Phys.). The relay will be manufactured by E. Leybold, Koln a/Rh. I was enabled to construct the apparatus by a grant of the Deutsche Forschungsgemeinschaft.

\author{
Physikalisches Institut der \\ Technischen Hochschule, \\ Dresden. \\ ${ }^{1}$ H. Greinacher, Helv. Phys. Acta, 7, 360 and $514 ; 1934.8,89$ \\ and $265 ; 1935$. Z. tech. Phys., 16, 165; 1935. \\ ${ }^{2}$ R. D. Summers, Rev. Sci. Inst., 6, 39; 1935.
}

\section{Effect of Light on Diamagnetic Susceptibilities}

THE effect of light on paramagnetic susceptibilities of the ions of the iron group has already been reported by Bose and Raha ${ }^{1}$. They have shown how the $l$-component of the magnetic moment, which is more or less quenched in these ions, is partially freed on exposure to light. We have since performed the corresponding experiment for some diamagnetics in a Curie-Cheneveau balance with a fine phosphor-bronze suspension.

The halogens in their ${ }^{1} \Sigma_{g}$ states are all known to be diamagnetic. Experiments with halogen vapours (chlorine, bromine and iodine) indicate that on exposure to light from which the infra-red portion was cut out, the susceptibilities increase in all cases. The exaltation of diamagnetism is evanescent at the red end, and remains very small until the violet end is reached. It is too early to offer a definite explanation, as the halogen molecule by absorbing light of wave-length shorter than the convergence limit (4785 A., 5107 A., 4995 A. for chlorine, bromine, iodine respectively) is adiabatically dissociated into two atoms $\left({ }^{2} P_{3 / 2}\right.$ and $\left.{ }^{2} P_{1 / 2}\right)$, both of which ought to be paramagnetic. This should result in a decrease, rather than an increase, of the average diamagnetic susceptibility of the vapour as a whole. But we may tentatively assume that the stationary concentration of atomic halogen is extremely small, and as such its effect is more than offset by the excited molecules to which the exaltation is due. The last we suggest in view of the fact that the effect, although small, is detectable even in the banded region.

The phenomenon is of the nature of a temporary shift of equilibrium only, as we could reproduce the dark deflection almost immediately the light was off. A side experiment with an air tube gave identical deflections both in the dark and in light.

$$
\begin{aligned}
& \text { Cotton College Laboratory, } \\
& \text { Gauhati, India. } \\
& { }^{2} \text { Bose and Raha, Phil. Mag., July } 1935 .
\end{aligned}
$$

says that "Evidence is available in Uganda which suggests the presence here of a large-flake culture, separate from, but contemporary with the Chellean".

If Mr. O'Brien is correct in his correlation and van Riet Lowe ${ }^{2}$ is correct in his classification of the Stellenbosch culture, according to which the Lower Stellenbosch uses "core and flake in almost equal proportions", then we must conclude that human history commenced at a very much later date in South than in East Africa.

Mr. O'Brien's correlation is, however, based purely on typology, deductions from which are vitiated by the inclusion of very serious assumptions in the line of reasoning, and Prof. Lowe's classification dates from a time when stratification of layers bearing various phases of the Stellenbosch culture, so far as natural deposits are concerned, were not known. Such a site is now known ${ }^{3}$ and the successive, stratified phases are :

(1) Small Clacton flakes. (2) Very large Clacton flakes made into very rough coups-de-poing and cleavers. (3) Smaller Clacton flakes used for making the coups-de-poing described by Mr. Goodwin ${ }^{4}$ from this area. A phase, apparently intermediate between $(2)$ and (3), was found in a raised beach (about 20 feet) a few miles away; and on the surface of eroded sites there was also: (4) Still smaller Clacton flakes and small, beautifully made coups-de-poing of the Fauresmith type. (5) A very numerous association of still smaller flakes, not reddened like those of phases (3) and (4) but with surfaces such as could have been formed by the action of wind-blown sand. No coups-de-poing could be found among these and typical levallois cores are very common. The use of cores is very rare in phase II, but the core technique is used to an increasing extent in phases III and IV.

We have thus at Mossel Bay an evolutionary series demanding as large a slice of time as can be demanded for the evolution of any similar series in any other part of the world.

When I mentioned, in a previous letter ${ }^{3}$, a Late Stellenbosch layer five or six feet above human remains in a shelter at Plettenberg Bay, I referred to a phase apparently intermediate between phases III and IV. I should perhaps have been more explicit than, for the sake of brevity, I was.

University College,

Bloemfontein, O.F.S. Oct. 20.

1 NATURE, 136, 475, Sept. 21, 1935.

S.A. J. Sci., 29, Oct. 1932 .

3 Roy. Soc. S.A., 22 , Pt. 3 ; 1934.

A Annals S.A. Mus., 27, and Roy. Soc. S.A., 16, Pt. 1.

S NATURE, 135, 620, April 20, 1935.

T. F. DREYER.

\section{Inhibitory Effect of Phloridzin on an Enzymic Dismutation}

RECENTLY it has been shown that phloridzin exerts an inhibitory effect on phosphorylations and dephosphorylations ${ }^{1}$. The action on enzymes other than diastase has not yet been investigated. On diastase no poisonous action of phloridzin was demonstrable ${ }^{1},{ }^{2}$; however, phloridzin is not a specific inhibitor of phosphorylation and dephosphorylation. As the present communication shows, the dis. mutative conversion of triosephosphoric acid into phosphoglyceric acid and glycerophosphoric acid is also prevented or markedly inhibited by phloridzin in concentrations of about $N / 100$. 